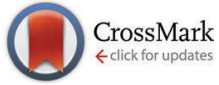

Cite this: Chem. Commun., 2014, 50, 12150

Received 29th July 2014, Accepted 19th August 2014

DOI: $10.1039 / \mathrm{c} 4 \mathrm{cc} 05867 d$

www.rsc.org/chemcomm

\section{Chemical modification of temoporfin - a second generation photosensitizer activated using upconverting nanoparticles for singlet oxygen generation $\dagger$}

\author{
Q. Yu, ${ }^{a}$ E. M. Rodriguez, ${ }^{a}$ R. Naccache, ${ }^{a}$ P. Forgione, ${ }^{a}$ G. Lamoureux, ${ }^{a}$ \\ F. Sanz-Rodriguez, ${ }^{\text {b D. Scheglmann }}{ }^{\text {C }}$ and J. A. Capobianco*a
}

$\mathrm{LiYF}_{4}: \mathrm{Tm}^{3+} / \mathrm{Yb}^{3+}$ upconverting nanoparticles (UCNPs) were functionalized with the second generation photosensitizer 5,10,15,20-tetra( $m$-hydroxyphenyl)chlorin ( $m$-THPC, Temoporfin, Foscan ${ }^{\circledR}$ ). $m$-THPC was modified using 4-(bromomethyl)benzoic acid, which induced a bathochromic shift of the $m$-THPC blue absorption peak. The nanoconstruct causes up to $70 \%$ cell death under $980 \mathrm{~nm}$ irradiation.

In photodynamic therapy (PDT), the destruction of a target tissue (localized tumor) involves the combination of light of a specific wavelength, oxygen and a photosensitizer (PS). ${ }^{1-3}$ Individually, these components are harmless; however, when combined, a putative cytotoxic agent, singlet oxygen $\left({ }^{1} \mathrm{O}_{2}\right)$, is generated. It is the ${ }^{1} \Delta_{\mathrm{g}}$ form of singlet oxygen that induces oxidation damages on cell components, resulting in cytotoxic reactions in the cells via necrosis or apoptosis, the self-destruction mechanisms of cells.

The second generation PS, 5,10,15,20-tetra( $m$-hydroxyphenyl)chlorin $^{4-6}$ ( $m$-THPC, Foscan ${ }^{\circledR}$, Temoporfin), with enhanced absorption of light in the red region $\left(\lambda_{\max }=652 \mathrm{~nm}\right)$ has been found to be more specific than conventional porphyrins where skin photosensitization, usually a side effect of PDT, has been reported to be shorter. However, $m$-THPC does not make use of the optimal spectral biological window for tissue penetration in the 700-1000 nm range. Unfortunately, photons in this region are energetically too low for ${ }^{1} \mathrm{O}_{2}$ generation. In addition to the band at $652 \mathrm{~nm}$, which has a high molar extinction coefficient $\left(\varepsilon=2.9 \times 10^{4} \mathrm{~cm}^{-1} \mathrm{M}^{-1}\right), m$-THPC has another absorption band in the blue region centered at $417 \mathrm{~nm}$ with a much higher molar absorption coefficient $\left(\varepsilon=2.3 \times 10^{5} \mathrm{~cm}^{-1} \mathrm{M}^{-1}\right)$. Excitation of $m$-THPC at $417 \mathrm{~nm}$ would eliminate the problem of low photon energy; however, direct excitation with high-energy blue light limits the use in biological applications due to its low tissue penetration depth. ${ }^{7}$

\footnotetext{
${ }^{a}$ Department of Chemistry and Biochemistry, Concordia University, Montreal, Canada. E-mail:John.Capobianco@concordia.ca

${ }^{b}$ Departamento de Biologia, Facultad de Ciencias, Universidad Autonoma de Madrid, Spain

${ }^{c}$ Research and Development, Biolitec AG, Germany

$\dagger$ Electronic supplementary information (ESI) available: Complete experimental and supplementary figures. See DOI: 10.1039/c4cc05867d
}

Lanthanide-doped upconverting nanoparticles ( $\mathrm{Ln}^{3+}$-UCNPs) have been intensively studied in recent years. These nanoparticles can emit UV, visible and/or near-infrared (NIR) light upon NIR excitation (typically $980 \mathrm{~nm}$ ) via a multiphoton process known as upconversion. The application of $\mathrm{Ln}^{3+}$-UCNPs as an energy source for exciting photosensitizers has been proposed. ${ }^{8-13}$

The nanoconstructs, which were prepared using upconverting nanoparticles for the excitation of different photosensitizers, made use of the $\mathrm{Er}^{3+}$ transitions, ${ }^{2} \mathrm{H}_{11 / 2},{ }^{4} \mathrm{~S}_{3 / 2} \rightarrow{ }^{4} \mathrm{I}_{11 / 2}$ and ${ }^{4} \mathrm{~F}_{9 / 2} \rightarrow{ }^{4} \mathrm{I}_{11 / 2}$. The transitions have one major disadvantage, the emission from these transitions does not overlap with the strongest absorption peak, the Soret band, of the photosensitizer presently used, which is at $\sim 400 \mathrm{~nm} .^{14}$ The ideal modification would be to shift the Soret band of the photosensitizer to obtain the maximum overlap with the emission band(s) of the $\mathrm{Ln}^{3+}$-UCNPs. Temoporfin is one example of an approved second-generation photosensitizer. ${ }^{15}$

Our group has recently synthesized $\mathrm{LiYF}_{4}: \mathrm{Tm}^{3+} / \mathrm{Yb}^{3+}$-UCNPs that showed stronger UV/blue emission following NIR excitation with $980 \mathrm{~nm}$ light compared to $\mathrm{NaYF}_{4}{ }^{16}$ Taking advantage of the high penetration depth and minimal autofluorescence of the NIR excitation light, $\mathrm{LiYF}_{4}: \mathrm{Tm}^{3+} / \mathrm{Yb}^{3+}$-UCNPs can be used as $\mathrm{UV} /$ blue excitation sources in biological applications.

In this study we report on the functionalization of $\mathrm{LiYF}_{4}: \mathrm{Tm}^{3+}$ / $\mathrm{Yb}^{3+}$-UCNPs with $m$-THPC to demonstrate the generation of singlet oxygen by this nanoconstruct using the blue light obtained following NIR excitation of the nanoparticles. In order to generate singlet oxygen from the upconverted blue light emission of the $\mathrm{LiYF}_{4}: \mathrm{Tm}^{3+} / \mathrm{Yb}^{3+}$-UCNPs, it was necessary to modify $m$-THPC using 4-(bromomethyl)benzoic acid (BMBA), which facilitated grafting of $m$-THPC onto the $\mathrm{LiYF}_{4}: \mathrm{Tm}^{3+} / \mathrm{Yb}^{3+}$-UCNPs, and induced a bathochromic shift of the $m$-THPC blue absorption peak. This produced an overlap with the emission peak(s) of the $\mathrm{LiYF}_{4}: \mathrm{Tm}^{3+} / \mathrm{Yb}^{3+}$-UCNPs that is of paramount importance for the efficient fluorescence resonance energy transfer.

The UCNPs utilized in this study, $\mathrm{LiYF}_{4}: \mathrm{Tm}^{3+} / \mathrm{Yb}^{3+}$, were synthesized via the thermal decomposition method. ${ }^{13}$ The resulting $\mathrm{LiYF}_{4}: \mathrm{Tm}^{3+} / \mathrm{Yb}^{3+}$-UCNPs showed a diamond-like morphology as evidenced by TEM (Fig. S1, ESI $\dagger$ ), and an average size of circa 
$86 \mathrm{~nm}$ along the long axis with an aspect ratio of 1.7. The upconversion luminescence spectrum of the oleate capped nanoparticles, upon irradiation with $980 \mathrm{~nm}$ light is shown in Fig. S2 (ESI $\dagger$ ).

We employed an acid treatment developed in our laboratory to remove the oleate ligand from their surface, ${ }^{17}$ which facilitates the direct conjugation of electronegative groups rendering the $\mathrm{LiYF}_{4}: \mathrm{Tm}^{3+} / \mathrm{Yb}^{3+}$-UCNPs amenable to $m$-THPC functionalization. In addition, $m$-THPC required modification to introduce an appropriate negatively charged group to bind to the positively charged surface of ligand-free $\mathrm{LiYF}_{4}: \mathrm{Tm}^{3+} / \mathrm{Yb}^{3+}$-UCNPs. In order to modify $m$-THPC, 4-(bromomethyl)benzoic acid (BMBA) was used as it is a commonly used "linker" in biological applications that is generally recognized as non-harmful to tissues and organs and provides the required carboxylate functionality to bind to the surface of the nanoparticles (Scheme S1, ESI $\dagger$ ). The coupling reaction of $m$-THPC and 4-(bromomethyl)benzoic acid produced a green colored product ( $m$-THPC-MBA) and UV/Vis analysis was carried out, and results were compared with those obtained using the unsubstituted parent (Fig. 1A). This showed four distinct differences, namely a red shift of the Soret band from $417 \mathrm{~nm}$ to $438 \mathrm{~nm}$, a lower molar absorption coefficient $(\varepsilon=1.05 \times$ $10^{4} \mathrm{~cm}^{-1} \mathrm{M}^{-1}$ ), peak broadening, and the disappearance of two of the Q-bands in the $500 \mathrm{~nm}$ region and the peak at $650 \mathrm{~nm}$.

Importantly, the number of linkers binding to the $m$-THPC and their position(s) may shed light on the possible reason(s) for the observed spectral differences. The LC-MS results (Fig. S3A, $\mathrm{ESI} \dagger$ ) showed that the product is a mixture consisting of three different compounds with 1 to 3 methyl benzoic acids bound to $m$-THPC and it was determined by chromatography that the major product has one linker bound to the $m$-THPC and the two minor products have two and three "linkers", respectively bound to $m$-THPC. The major product was isolated and characterized by LC-MS and demonstrated two main peaks, each showing a molecular ion peak at 815 (Fig. S3B, ESI $\dagger$ ).

UV/Vis absorption spectra (Fig. 1B) of the two eluted fractions were obtained and the fraction that has an elution time of $10.1 \mathrm{~min}$ demonstrated excellent correspondence with the absorption spectrum of $m$-THPC, whereas the fraction with an elution time of 7.5 min showed a red shift in comparison to $m$-THPC.

Although aggregation of $m$-THPC has been proposed to occur in polar and/or aqueous solvents, ${ }^{18-20}$ this typically occurs

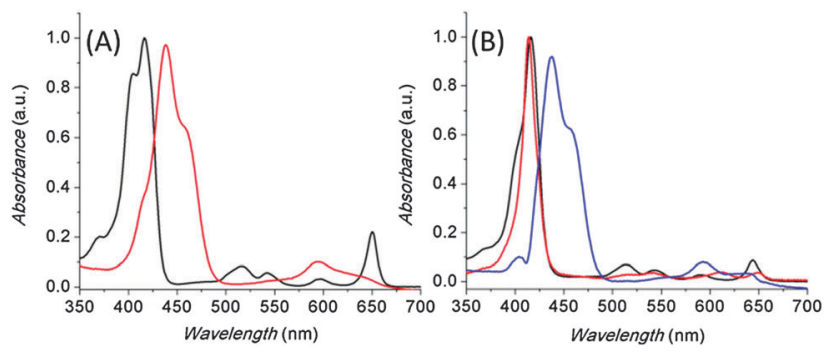

Fig. 1 (A) UV/Vis spectra of (-) m-THPC and (-) the m-THPC-MBA product. (B) UV/Vis absorption spectra of $(-) m$-THPC, $(-) m$-THPC$\mathrm{MBA}(\mathrm{I})$ and (॥) isomers eluted at $10.1 \mathrm{~min}$ and (-) $m$-THPC-MBA(II) isomers eluted at $7.5 \mathrm{~min}$ from Prep LC-MS. All the spectra were measured in the ethanol-propylene glycol mixture solvent. when $\left[m\right.$-THPC] is greater than $10^{-4} \mathrm{M}$. As our studies were conducted at $10^{-5} \mathrm{M}$ this excludes the possibility that the red shift may be due to aggregation. Alternatively, the red shift could be caused by the addition of methyl benzoic acid to the $m$-THPC that serves as an electron-donating group, increasing the electron density and lowering the energy gap between the ground and excited state resulting in absorption at a longer wavelength.

It has been well documented that changes in the conjugation pathway and symmetry of porphyrin can affect its UV/Vis absorption spectrum. The absorption spectrum of porphyrin has long been understood by the four orbital model first applied by Gouterman. ${ }^{21,22}$ The method demonstrated the importance of charge localization on electronic spectroscopic properties. Density functional theory and time-dependent methods have been employed successfully to explore the electronic structure and spectral properties of porphyrin and related compounds. ${ }^{23,24}$ Thus, to understand the observed spectral changes (effects of the substituent and its position) we undertook a computational study using the Gaussian 09 program. $^{25}$

The calculations ( $m$-THPC) show that the chlorin macrocycle is planar and the four phenyl groups at positions 5, 10, 15, 20 do not lie planar to the chlorin macrocycle, but are positioned at an angle of $60^{\circ}$ (Fig. S4A, ESI $\dagger$ ). It has been previously shown that this geometrical arrangement of the phenyl groups is responsible for only a minor shift of the absorption bands compared to chlorin itself. ${ }^{26}$ For the modified $m$-THPC-MBA(I) and $m$-THPC-MBA(II) where the methyl benzoic acid linker is added to the phenyl groups at positions 5 and 20, the linker is at an angle of $27^{\circ}$ from the $x y$ plane and parallel to the chlorin macrocycle, respectively (Fig. S4B, ESI $\dagger$ ). For $m$-THPC-MBA(III) where the linker is added to the nitrogen in the chlorin ring, it was found to be at an angle of $67^{\circ}$ to the chlorin macrocycle. More importantly, the two nitrogens of the pyrrole move upwards from the $x y$ plane and as a consequence the chlorin ring is distorted, and thus the symmetry of the macrocycle is broken.

The chlorin macrocycle is the reduced form of porphyrin, thus Gouterman's four-orbital model was applied to calculate the absorption spectra of $m$-THPC and of the three isomers of $m$-THPC-MBA. The absorption bands in chlorin arise from transitions between two HOMOs and two LUMOs. Fig. S5 (ESI $\dagger$ ) shows the calculated molecular orbitals from HOMO -1 to $\mathrm{LUMO}+1$ for $m$-THPC and the three isomers. In Table S1 (ESI $\dagger$ ), the calculated excitation energies and the oscillator strengths in ethanol are shown. The results reveal a bathochromic shift in the Soret band of $m$-THPC-MBA(III) compared with the excitation energies calculated for $m$-THPC and $m$-THPC-MBA(I, II). The calculated electronic absorption spectra (in ethanol) of the three isomers and $m$-THPC are shown in Fig. 2A.

The linker MBA is an electron donor, thus we would expect a change in electron density in the temoporfin molecule, which may affect the absorption spectrum. However, the simulation results for the two isomers $m$-THPC-MBA(I, II) clearly show that the addition of MBA has no effect on the absorption properties. The linker is $6.16 \AA$ from the chlorin ring, in addition there is an isolating group, $-\mathrm{CH}_{2}$, between the carboxyphenyl and the 

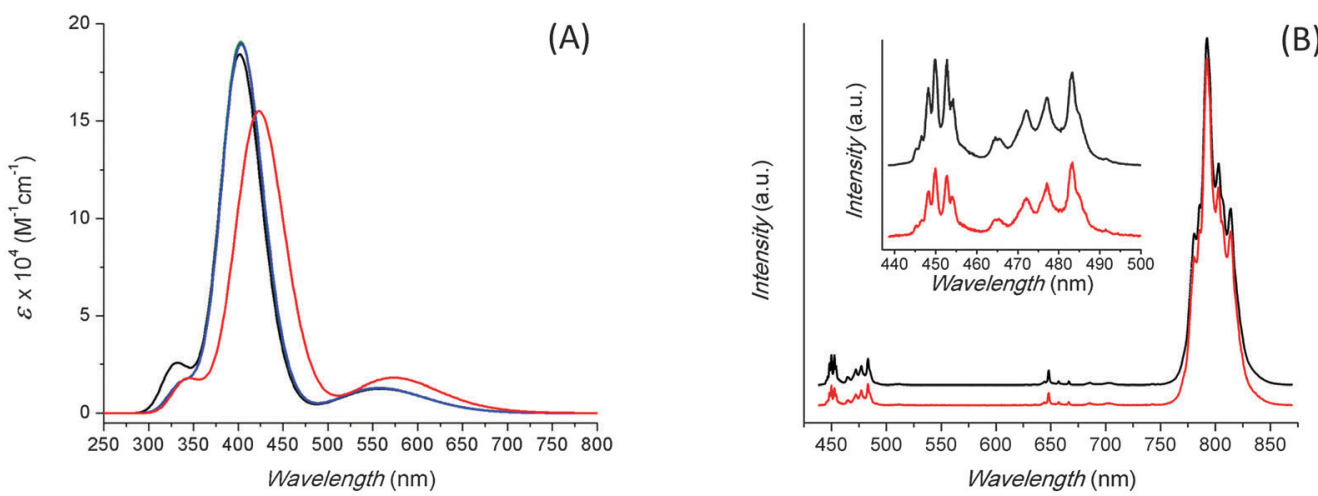

Fig. 2 (A) The simulated absorption spectra of $(-) m$-THPC, ( - $m$-THPC-MBA(I), ( - $m$-THPC-MBA(॥) and (-) $m$-THPC-MBA(॥) calculated by TD-DFT/B3LYP in ethanol using CPCM solvation. The spectra of $m$-THPC-MBA $(1)$ and $m$-THPC-MBA(॥) are indeed overlapping. (B) Upconverting emission spectra demonstrating the energy transfer between the $\mathrm{Ln}^{3+}$-UCNPs and the photosensitizer. A decrease of intensity was observed in the blue region upon comparing the (一) oleate-free $\mathrm{LiYF}_{4}: \mathrm{Tm}^{3+} / \mathrm{Yb}^{3+}-\mathrm{UCNPs}$ with (-) m-THPC-MBA-LiYF $4: \mathrm{Tm}^{3+} / \mathrm{Yb}^{3+}-\mathrm{UCNPs}$.

phenyl ring, therefore the linker is not conjugated to the chlorin ring and has no influence on the absorption properties. In contrast, the addition of the linker on the nitrogen in the chlorin ring results in a bathochromic shift of the Soret band. In this case, the proximity of methyl benzoic acid to the conjugated system increases the electron density, resulting in a decrease of the energy gaps between the HOMO and the LUMO, which manifests itself as a red shift of the Soret band. The distortion of the chlorin ring caused by the addition of the MBA may also contribute to the bathochromic shift. The computational results have allowed us to determine $m$-THPCMBA(III) to be the isolated product, which is responsible for the red shift of the Soret band from $417 \mathrm{~nm}$ to $438 \mathrm{~nm}$.

The upconversion emission spectra of a dispersion of oleatefree $\mathrm{LiYF}_{4}: \mathrm{Tm}^{3+} / \mathrm{Yb}^{3+}$-UCNPs $(1 \mathrm{wt} \%$ in ethanol-propylene glycol mixture) and of a solution of $m$-THPC-MBA-LiYF $4: \mathrm{Tm}^{3+} / \mathrm{Yb}^{3+}$ UCNPs ( $1 \mathrm{wt} \%$ in the ethanol-propylene glycol mixture) are shown in Fig. 2B. We observe a decrease in the intensity of the blue peaks for the $m$-THPC-MBA-LiYF $4: \mathrm{Tm}^{3+} / \mathrm{Yb}^{3+}$-UCNPs in comparison to the respective peaks for the oleate-free $\mathrm{LiYF}_{4}: \mathrm{Tm}^{3+}$ / $\mathrm{Yb}^{3+}$-UCNPs.

The spectra were normalized with respect to the NIR emission peak at $800 \mathrm{~nm}$ and the ratio of the intensity of the blue emission bands for the solution of oleate-free-LiYF ${ }_{4}: \mathrm{Tm}^{3+} / \mathrm{Yb}^{3+}$ UCNPs and $m$-THPC-MBA-LiYF $4: \mathrm{Tm}^{3+} / \mathrm{Yb}^{3+}$-UCNPs was found to be $3: 2$. This indicates that the binding of $m$-THPC-MBA on the UCNPs resulted in the quenching of the emission of the blue peaks, which clearly demonstrates that resonance energy transfer from the $\mathrm{LiYF}_{4}: \mathrm{Tm}^{3+} / \mathrm{Yb}^{3+}$-UCNPs to $m$-THPC is occurring. The energy transfer efficiency was calculated to be $\sim 33 \%$. These results also confirm that the spatial separation, which was calculated to be $7 \AA$ between the donor and the acceptor molecule, is small enough to favor non-radiative energy transfer from the nanoparticle to the photosensitizer. The number of $m$-THPCMBA molecules bound to each UCNP was found to be $7.6 \times 10^{3}$.

The generation of ${ }^{1} \mathrm{O}_{2}$ is primordial in photodynamic therapy, thus we assessed its production by the nanoparticle photosensitizer construct ( $m$-THPC-MBA-LiYF $4: \mathrm{Tm}^{3+} / \mathrm{Yb}^{3+}$-UCNPs) under $980 \mathrm{~nm}$ excitation using the standard reaction of 1,3-diphenylisobenzofuran (DPBF) with singlet oxygen. ${ }^{27,28}$ We exposed a solution of DPBF and $m$-THPC-MBA-LiYF $4: \mathrm{Tm}^{3+} / \mathrm{Yb}^{3+}$-UCNPs in an ethanolpropylene glycol mixture to an irradiation source of $980 \mathrm{~nm}$ over a period of $120 \mathrm{~min}$ at 10 minute intervals. Fig. S6 (ESI $\dagger$ ) shows that the absorbance at $415 \mathrm{~nm}$ of DPBF decreases as the irradiation time increases, which indicates that the amount of singlet oxygen production increases. The concentration of singlet oxygen generated was calculated from the consumption of DPBF and it was found to be $2.11 \times 10^{-5} \mathrm{M}$.

In vitro cell viability tests were performed to confirm the effect of the nanoconstruct on the cells in the absence and presence of irradiation at $980 \mathrm{~nm}$. We also confirmed that the UCNPs, which were not functionalized with $m$-THPC-MBA did not cause cell death (Fig. 3A). HeLa carcinoma cells were incubated for 4 hours with the nanoconstruct ( $m$-THPC-MBA-LiYF ${ }_{4}: \mathrm{Tm}^{3+} / \mathrm{Yb}^{3+}$-UCNPs) at concentrations of 0.004 to $0.1 \mathrm{mg} \mathrm{mL}^{-1}$ in PBS. Cell viability was assayed using the MTT assay, a method based on the activity of mitochondrial dehydrogenase. ${ }^{29}$ The nanoconstruct demonstrated no intrinsic toxicity towards the cells when not irradiated, as the cellular survival rate was identical to that of the control (Fig. 3A). To assess the efficacy of the photodynamic effect, HeLa cells were incubated for 4 hours with the nanoconstruct at the same concentration as stated above, which represents a concentration of 0.10 to $2.50 \mu \mathrm{M}$ of $m$-THPC-MBA and irradiated for 1 hour using NIR radiation ( $980 \mathrm{~nm}$ ). Cell death was observed from a minimum of $20 \%$ to a maximum of $70 \%$ in the concentration range $0.50-2.50 \mu \mathrm{M} m$-THPC-MBA (Fig. 3A). Such a significant cell death demonstrates the possible applicability of the nanoconstructs in photodynamic therapy using NIR irradiation.

In conclusion, we have successfully prepared the nanoconstruct shown in Fig. 3B based on modified Temoporfin (m-THPC-MBA) grafted on a carrier nanoparticle $\mathrm{LiYF}_{4}: \mathrm{Tm}^{3+} / \mathrm{Yb}^{3+}$ using a method that permits the photosensitizer to be functionalized directly to the $\mathrm{LiYF}_{4}: \mathrm{Tm}^{3+} / \mathrm{Yb}^{3+}$-UCNPs. The computational results allowed us to determine that $m$-THPC-MBA(III) is the isolated product, which is responsible for the red shift of the Soret band from $417 \mathrm{~nm}$ to $438 \mathrm{~nm}$ and ascertain that the linker is 


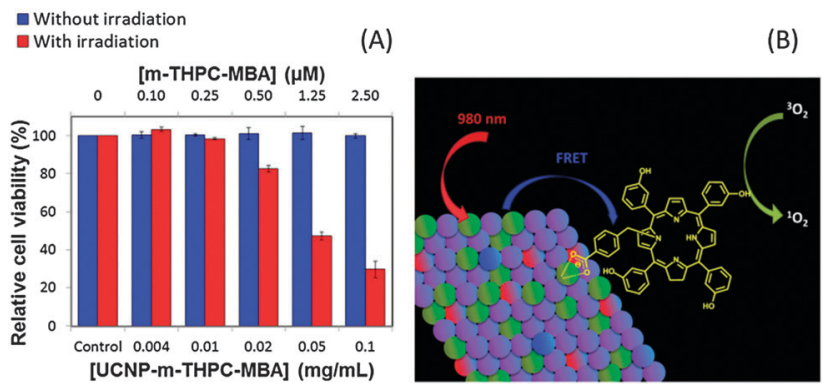

Fig. 3 (A) Viability of HeLa cancer cells following incubation with the nanoconstructs and in the presence and absence of NIR irradiation (1 hour irradiation period). The bottom axis shows the concentration of the nanoconstruct, while the top axis shows the equivalent concentration with respect to the PDT agent. (B) Schematic illustration showing the nanoconstruct synthesized from $\mathrm{LiYF}_{4}: \mathrm{Tm}^{3+} / \mathrm{Yb}^{3+}$ nanoparticles and photosensitizer $m$-THPC-MBA(III). The diamond is representing the surface of the nanoparticle: purple spheres $=$ $\mathrm{F}^{-}$, green spheres $=\mathrm{Y}^{3+}$, red spheres $=\mathrm{Yb}^{3+}$, and blue spheres $=\mathrm{Tm}^{3+}$. The carboxylate from $m$-THPC-MBA(III) is coordinated to the $\mathrm{Y}^{3+}$ ion of the $\mathrm{Ln}^{3+}-\mathrm{UCNP}$ via electrostatic interaction.

bonded to the nitrogen of the pyrrole ring. The photosensitizer can be excited by irradiation of the $\mathrm{LiYF}_{4}: \mathrm{Tm}^{3+} / \mathrm{Yb}^{3+}$-UCNPs at $980 \mathrm{~nm}$ to produce ${ }^{1} \mathrm{O}_{2}$. This demonstrates that the $m$-THPC-MBA molecules on the surface of the $\mathrm{LiYF}_{4}: \mathrm{Tm}^{3+}$ / $\mathrm{Yb}^{3+}$-UCNPs are excited via an energy transfer process and are capable of performing PDT. More importantly, using an irradiation wavelength of $980 \mathrm{~nm}$ would enable PDT in "deep" tissues. We also demonstrated that the nanoconstruct was capable of causing cell death up to $70 \%$ under $980 \mathrm{~nm}$ irradiation for a period of 1 hour. The general method reported can be applied to other drugs for the preparation of nanoparticles as drug carriers.

J.A.C. is a Concordia University Research Chair in Nanoscience and is grateful to Concordia University for financial support. J.A.C., P.F., G.L. and R.N. are grateful for the support from (NSERC) Canada.

\section{Notes and references}

1 R. L. Morris, K. Azizuddin, M. Lam, J. Berlin, A. Nieminen, M. E. Kenney, A. C. S. Samia, C. Burda and N. L. Oleinick, Cancer Res., 2003, 63, 5194.
2 R. Bonnett, Chem. Soc. Rev., 1995, 24, 19.

3 T. J. Dougherty, C. J. Gomer, B. W. Henderson, G. Jori, D. Kessel, M. Korbelik, J. Moan and Q. Peng, J. Natl. Cancer Inst., 1998, 90, 889.

4 J. C. M. Stewart, Curr. Opin. Invest. Drugs (BioMed Cent.), 1993, $2,1279$.

5 S. Mitra and T. H. Foster, Photochem. Photobiol., 2005, 81, 849.

6 A. Zimmermann, M. Ritsch-Marte and H. Kostron, Photochem. Photobiol., 2001, 74, 611.

7 Á. Juarranz, P. Jaén, F. Sanz-Rodríguez, J. Cuevas and S. González, Clin. Transl. Oncol., 2008, 10, 148.

8 Y. I. Park, H. M. Kim, J. H. Kim, K. C. Moon, B. Yoo, K. T. Lee, N. Lee, Y. Choi, W. Park, D. Ling, K. Na, W. K. Moon, S. H. Choi, H. S. Park, S. Y. Yoon, Y. D. Suh, S. H. Lee and T. Hyeon, Adv. Mater., 2012, 24, 5755.

9 P. Zhang, W. Steelant, M. Kumar and M. Scholfield, J. Am. Chem. Soc., 2007, 129, 4526.

10 H. S. Qian, H. C. Guo, P. C.-L. Ho, R. Mahendran and Y. Zhang, Small, 2009, 5, 2285.

11 B. Ungun, R. K. Prud'homme, S. J. Budijono, J. Shan, S. F. Lim, Y. Ju and R. Austin, Opt. Express, 2009, 17, 80.

12 K. Liu, X. Liu, Q. Zeng, Y. Zhang, L. Tu, T. Liu, X. Kong, Y. Wang, F. Cao, S. A. G. Lambrechts, M. C. G. Aalders and H. Zhang, ACS Nano, 2012, 6, 4054.

13 S. Cui, D. Yin, Y. Chen, Y. Di, H. Chen, Y. Ma, S. Achilefu and Y. Gu, ACS Nano, 2013, 7, 676.

14 M. C. DeRosa and R. J. Crutchley, Coord. Chem. Rev., 2002, 233-234, 351.

15 S. Banfi, E. Caruso, S. Caprioli, L. Mazzagatti, G. Canti, R. Ravizza, M. Gariboldi and E. Monti, Bioorg. Med. Chem., 2004, 12, 4853.

16 V. Mahalingam, F. Vetrone, R. Naccache, A. Speghini and J. A. Capobianco, Adv. Funct. Mater., 2009, 21, 4025.

17 N. Bodgan, F. Vetrone, G. Ozin and J. A. Capobianco, Nano Lett., 2011, 11, 835.

18 R. Bonnett, B. D. Djelal and A. Nguyen, J. Porphyrins Phthalocyanines, 2001, 5, 652.

19 R. Bonnett, P. Charlesworth, B. D. Djelal, S. Foley, D. J. McGarvey and T. G. Truscott, J. Chem. Soc., Perkin Trans. 2, 1999, 325.

20 F. Würthner, T. E. Kaiser and C. R. Saha-Möller, Angew. Chem., Int. Ed., 2011, 50, 3376.

21 M. Gouterman, J. Mol. Spectrosc., 1961, 6, 138.

22 M. Gouterman, J. Chem. Phys., 1959, 30, 1139.

23 E. S. E. Eriksson and L. A. Eriksson, Phys. Chem. Chem. Phys., 2011, 13, 7207.

24 R. E. Stratmann, G. E. Scuseria and M. J. Frisch, J. Chem. Phys., 1998, 109, 8218.

25 M. J. Frisch, et al., Gaussian 09, Revision B.01, Gaussian, Inc., Wallingford CT, 2009.

26 E. S. E. Eriksson and L. A. Eriksson, Phys. Chem. Chem. Phys., 2011, 13, 11590.

27 C. Hadjur, N. Lange, J. Rebstein, P. Monnier, H. Bergh and G. Wagnières, J. Photochem. Photobiol., B, 1998, 45, 170.

28 W. Spiller, H. Kliesch, D. Wöhrele, S. Hackbarth, B. Röder and G. Schnurpfeil, J. Porphyrins Phthalocyanines, 1998, 2, 145.

29 M. V. Berridge and A. S. Tan, Arch. Biochem. Biophys., 1993, 303, 474. 